\title{
OBSERVATIONS OF SOUTHERN DWARF NOVAE
}

\section{N. Vogt}

European Southern Observatory

Preliminary results of spectroscopic and photometric data for five dwarf novae are presented:

1) V 436 Cen. The orbital period of 0.0669 days was determined from radial velocity variations. The RV half amplitude of the primary, $K_{1}=159 \mathrm{~km} / \mathrm{s}$, implies very small masses of $M_{1} \leqslant 0.20 M_{0}$ and $M_{2}=$ $0.18 \mathrm{M}_{\odot}$ for the binary components.

2) $Z$ Cha. Broad emission ( $\mathrm{H} \beta, \mathrm{H} \gamma, \mathrm{H} \delta$ ) and superimposed narrow absorption lines of $\mathrm{HB}-\mathrm{H} 11$, HeI 4471 , CaI 4427 and CaII $\mathrm{K}$ characterize the spectrum during quiescence. Apparently, the cool, optically thin outer disc is seen on the background of a hot continuum, originating from the white dwarf or the Inner disc. The RV half amplitude $K_{1}=87 \mathrm{~km} / \mathrm{s}$ results in masses of $\mathrm{M}_{1}=1.10 \mathrm{M}_{0}$ and $\mathrm{M}_{2}=0.21 \mathrm{M}_{\odot}$.

3) EX Hya. The RV half amplitude $K_{1}=68 \mathrm{~km} / \mathrm{s}$ reveals masses of $M_{1}=$ $\overline{1.4 \mathrm{M}_{\odot}}$ and $\mathrm{M}_{2}=0.19 \mathrm{M}_{\odot}$. The equivalent widths of the emission lines of $\mathrm{H}, \mathrm{HeI} 4471$ and HeII 4686 vary with the phase of the recently detected 67 minute cycle (maximal $\mathrm{EW}$ coincides nearly with maximal continuum intensity).

4) OY Car is an eclipsing binary with an orbital period of 0.0631 days. The eclipses show strong variations in shape and amplitude in the course of an outburst, similar as those of $Z$ Cha. The observations seem to confirm that the location of the eruption is the central part of the disc which increases in size and luminosity.

5) EK TrA shows periodic superhumps ( $P=0.0645$ days) during supermaximum, and therefore belongs to the SU UMa sub-group of dwarf novae which are also characterized by a quasi-periodic occurence of supermaxima. The SU UMa sub-group comprises $70 \%$ of the ultra-short period cataclysmic binarles, and at least $18 \%$ of all dwarf novae. 